\title{
Aspectos de Segurança do Tratamento com hrGH
}

revisão

\section{CRISTIANE KOCHI}

Unidade de Endocrinologia
Pediátrica da Santa Casa de
São Paulo, SP, Brasil.
O tratamento com hormônio de crescimento recombinante humano ( $\mathrm{hrGH}$ ) tem sido realizado há mais de 20 anos, visto que seu perfil de segurança é considerado excelente. Nos principais bancos de dados internacionais, os eventos adversos relatados são raros, principalmente em pacientes com deficiência isolada do hormônio de crescimento e na baixa estatura idiopática. Em relação à associação com risco de malignidade ou de recorrência tumoral, os dados sugerem que não há maior incidência em pacientes em uso de hrGH do que na população geral. A hipertensão intracraniana benigna é rara, porém sua incidência é maior em pacientes com insuficiência renal crônica. Apesar de os eventos adversos serem raros, é importante manter a monitorização cuidadosa dos pacientes em uso do $\mathrm{hrGH}$, principalmente aqueles em uso de doses farmacológicas ou com patologias associadas que confiram maior risco de complicação. (Arq Bras Endocrinol Metab 2008; 52/5:850-853)

Descritores: Hormônio do crescimento/deficiência; Hormônio do crescimento/farmacologia; Hormônio do crescimento/uso terapêutico; Segurança; Eventos adversos

\section{ABSTRACT}

\section{Safety Aspects of Treatments with hrGH.}

Recombinant human growth hormone $(\mathrm{hrGH})$ has been used for treatment of growth hormone deficient children over 20 years and it can be considered to have an excellent safety profile. The main international surveys show few adverse drug reactions reported, specially in idiopathic growth hormone deficiency and in idiopathic short stature. With regard to cancer risk or tumor recurrence, it seems that there is no greater risk with hrGH treatment in comparison to general population. The idiopathic intracranial hypertension is a rare condition, but its incidence is higher in patients with chronic renal failure. The adverse events are rare in patients treated with $\mathrm{hrGH}$, but these patients should be carefully monitored, specially those with pharmacologycal doses or with another clinical condition of greater risk. (Arq Bras Endocrinol Metab 2008; 52/5:850-853)

Keywords: Growth hormone/deficiency; Growth hormone/pharmacology; Growth hormone/therapeutic use; Growth hormone/safety; Adverse events.

\section{INTRODUÇÃo}

Recebido em 16/4/2008

Aceito em 19/4/2008
HORMONNIO DE CRESCIMENTO recombinante humano (hrGH) foi disponibilizado no mercado a partir de 1985. Desde então, há grande preocupação das agências regulatórias em relação aos aspectos de segurança dessa medicação. 
A partir de sua comercialização, há pouco mais de 20 anos, o hrGH tem apresentado perfil de segurança excelente no tratamento de crianças com deficiência do hormônio de crescimento (DGH), com poucas reações adversas sendo relatadas (1). No entanto, o uso do hrGH vem se tornando mais amplo, incluindo situações nas quais não há deficiência de secreção do hormônio de crescimento, como as síndromes que cursam com baixa estatura (síndrome de Turner, síndrome de Noonan, síndrome de Prader-Willi, entre outras), insuficiência renal crônica, restrição de crescimento intrauterino e em pacientes adultos com DGH (2). Portanto, há necessidade de se continuar a monitoração da segurança do tratamento com hrGH.

\section{EVENTOS ADVERSOS}

O key indicators graphical system (KIGS) é um grande banco de dados de crianças tratadas com hrGH. Até janeiro de 2006, mais de 56 mil pacientes foram inclú́dos, representando 164.558 anos de tratamento com hrGH. Nesse período, foram relatados 16.971 eventos adversos. No entanto, a definição de evento adverso no KIGS inclui todas as doenças concomitantes que ocorreram durante o tratamento com hrGH (3). O National Cooperative Growth Study (NCGS) é outro grande banco de dados, com mais de 47 mil pacientes tratados e monitorados desde 1985, representando 165 mil pacientes-ano (4). Portanto, vários dados referidos a seguir são relacionados a esses dois bancos de dados multicêntricos.

\section{Risco de malignidade}

Desde o primeiro caso de leucemia descrito em paciente em uso de hrGH, muito se tem debatido em relação à essa associação. A incidência de leucemia em crianças com DGH não-tratadas não é conhecida. Alguns relatos de casos de leucemia em crianças com DGH nunca tratadas tem sugerido que o risco aumentado de desenvolver leucemia nesse grupo de pacientes é independente do tratamento com hrGH (5). A incidência de leucemia em crianças de até 15 anos de idade é aproximadamente de $1: 30.000$, dado semelhante ao observado no KIGS, sugerindo que não há aumento da incidência de leucemia em crianças tratadas com hrGH. Dados do NCGS até 2002 também não mostraram aumento de incidência de casos de leucemia ou de outros tumores (4).
Vários relatos têm demonstrado que o risco de recorrência tumoral ou de desenvolvimento de um segundo tumor não é maior em pacientes tratados com hrGH. No entanto, em recente publicação, ErgunLongmire e cols. descrevem risco maior de desenvolvimento de segunda neoplasia sólida em pacientes sobreviventes de câncer submetidos ao tratamento com hrGH, embora esse risco diminua com o tempo de acompanhamento e o risco geral seja pequeno (6).

Em pacientes com neoplasia que desenvolvem DGH secundária ao tumor ou ao seu tratamento, a época de início do hrGH deve ser individualizada, levando-se em consideração o tipo de tumor, o término do tratamento e o período de remissão (7).

Alguns grupos apresentam risco intrínseco maior de desenvolvimento de neoplasias, como a síndrome de Down, a síndrome de Bloom, a anemia de Fanconi e a neurofibromatose tipo 1 . Nesses casos, os pacientes devem ser cuidadosamente monitorados em relação ao aparecimento tumoral (7).

\section{Hipertensão intracraniana benigna}

A hipertensão intracraniana benigna ocorre em cerca de 1:1.000 pacientes tratados com hrGH, porém esse dado pode estar subestimado (8). Toda queixa de cefaléia deve ser valorizada e investigada. O fundo de olho deve ser realizado no início do tratamento e repetido quando houver indicação clínica (7).

No KIGS, a maior incidência de hipertensão intracraniana benigna ocorreu em pacientes com insuficiência renal crônica (3). Esses dados estão de acordo com os do NCGS, em que a maior incidência foi observada na insuficiência renal crônica, seguida pela síndrome de Turner em comparação aos pacientes com DGH e baixa estatura idiopática, porquanto nenhum caso foi relatado nessa última condição $(4)$.

\section{Diabetes melito}

Tem sido sugerido que o hrGH poderia causar alterações do metabolismo de carboidratos, visto que há alta incidência de diabetes melito em pacientes com acromegalia e pelo aumento da resistência insulínica causada pelo GH. No entanto, na maioria dos pacientes, a homeostase da glicose é mantida dentro do normal. O aumento de casos de diabetes tipo 1 ou 2 não parece ser maior do que na população em geral. Porém, um subgrupo de pacientes, como síndrome de Turner, síndrome de Prader-Willi e restrição do crescimento intra-uterino tem 
maior risco de evolução para diabetes, devendo ser monitorados cuidadosamente em relação ao metabolismo de carboidratos (7). $\mathrm{O}$ diabetes não é contra-indicação do uso do hrGH, visto que o controle glicêmico deve seguir as rotinas habituais de tratamento.

A concentração plasmática de insulina costuma estar aumentada tanto em jejum quanto após sobrecarga oral de glicose, mas esse aumento não piora após três anos de uso de hrGH. A hiperinsulinemia causada pelo tratamento com hrGH poderia, na verdade, amplificar a ação anabólica que a insulina normalmente promove no metabolismo protéico durante a puberdade (9).

\section{Alterações esqueléticas}

A incidência de escorregamento epifisário da cabeça do fềmur na população geral entre 7 e 17 anos é de cerca de 10 a 12 casos/100.000. Os prováveis fatores de risco associados são crescimento rápido, obesidade e hipotireoidismo. A incidência de escorregamento epifisário observada no KIGS foi de 19:100.000, ou seja, semelhante à população geral (3).

Não parece haver associação entre o escorregamento em pacientes em uso de hrGH e sexo, dose, tempo de tratamento ou rápido crescimento inicial. Crianças com baixa estatura idiopática são de menor risco do que as crianças com DGH, achado esse que pode sugerir a deficiência hormonal como condição predisponente (4).

Em relação à escoliose e à necrose avascular da cabeça do fêmur, não há evidências de que sejam causadas pelo tratamento com hrGH, porém pode haver piora da escoliose quando o crescimento é acelerado (7).

\section{Convulsões}

A incidência de convulsões, no KIGS, variou de acordo com o subgrupo de pacientes. Nos pacientes com DGH isolada, síndrome de Turner e baixa estatura idiopática, a incidência foi semelhante à da população geral. Já houve maior incidência em pacientes com tratamento prévio para tumor de sistema nervoso central (SNC). É possível que a neurocirurgia ou a radioterapia de SNC possam reduzir o limiar para convulsão (3).

\section{Interação com outros hormônios}

Não há nenhuma evidência de que o hrGH possa ter algum evento adverso no desenvolvimento puberal ou na função gonadal. Já no metabolismo tireoidiano, o hrGH aumenta a conversão extratireoidiano de T4 em T3, podendo deixar evidente o hipotireoidismo inci- piente. Portanto, a monitoração da função tireoidiana deve ser realizada em todos os pacientes (7). O hrGH diminui a concentração plasmática de cortisol por reduzir a concentração da globulina ligadora do cortisol (CBG). Além disso, também pode reduzir a biodisponibilidade do cortisol por permitir maior conversão de cortisol em cortisona. Apesar de a implicação clínica dessas alterações ainda não estar bem estabelecida, recomenda-se monitorar o status do cortisol nos pacientes em tratamento com hrGH (7).

\section{Pacientes com doença grave}

$\mathrm{O}$ uso de doses farmacológicas de hrGH em pacientes graves está associado à maior morbimortalidade. Portanto, atualmente recomenda-se não iniciar tratamento com doses farmacológicas em pacientes graves e suspender o tratamento quando o paciente já está em uso de doses farmacológicas e evolui com doença aguda grave.

Em relação a doses habituais de hrGH, não há dados suficientes para indicar ou não a interrupção do tratamento normal de reposição com hrGH em pacientes que evoluem com quadro grave ou são submetidos à cirurgia (7).

De acordo com os subgrupos de patologias tratadas com hrGH, observou-se menor incidência de eventos adversos na DGH idiopática em comparação à DGH congênita, sendo nessa última a incidência de hipertensão intracraniana benigna quatro vezes maior. Nos pacientes com craniofaringioma também foi notada maior incidência de eventos adversos, com 13\% de recorrência do craniofaringioma após período médio de tratamento de 1,9 anos (3). Na insuficiência renal, houve maior incidência de hipertensão intracraniana benigna, escoliose e convulsão do que qualquer outro subgrupo. Na síndrome de Prader-Willi, as principais complicações estiveram relacionadas à escoliose (27 vezes mais freqüente) e diabetes melito (19 vezes mais freqüente que a DGH idiopática). O grupo da baixa estatura idiopática foi o de menor incidência de eventos adversos (3).

\section{UTILIZAÇÃO DA rhIGF-1}

Os pacientes com insensibilidade ao hormônio de crescimento são tratados com rhIGF-1, que é efetiva em promover o crescimento dessas crianças. No primeiro ano de tratamento, há aumento importante da velocidade de crescimento, porém esses pacientes não atingem seu potencial genético de estatura. Um dos motivos seria o fato de a rhIGF-1 ter meia-vida mais curta, pois esses pacien- 
tes apresentam concentrações plasmáticas reduzidas de IGFBP-3 e da subunidade ácido lábil. No estudo de acompanhamento de 76 pacientes por até 12 anos de tratamento, mostrou que $49 \%$ deles apresentaram um ou mais episódios de hipoglicemia durante o período de tratamento, com $5 \%$ de crises convulsivas. Outros efeitos relatados foram: hipoacusia, hipertrofia de adenóide (22\%), hipertrofia do timo (35\%), lipohipertrofia no local da injeção (32\%), queixas ortopédicas (20\%) (10). Com o intuito de aumentar a meia-vida plasmática da IGF-1 e reduzir seus efeitos colaterais, foi desenvolvida uma mistura equimolar de IGF-1 e IGFBP-3 (rinfabato de mecasermina). Estudos têm mostrado menor incidência de hipoglicemia com esse preparado (11). Porém, em recente levantamento bibliográfico a respeito de sua farmacocinética e efeitos colaterais, foi observado que o rinfabato de mecasermina tem efeitos biológicos muito parecidos aos da rhIGF-1, assim como seus eventos adversos. Ainda há poucos dados publicados sobre a farmacocinética desse medicamento em humanos (12).

\section{MONITORIZAÇÃO}

Pacientes em uso do hrGH devem ser monitorizados com dosagens séricas de IGF-1, IGFBP-3, glicemia e insulinemia, além dos aspectos clínicos.

Relatos a partir de casos de acromegalia têm sugerido que concentrações elevadas de IGF-1 e baixas de IGFBP-3 estão associadas ao maior risco de câncer, especialmente de cólon, próstata e mama $(13,14)$. Park e Cohen têm sugerido que em crianças os valores de IGF-1 devem ser relacionados aos valores de IGFBP-3 (15). Estudos utilizando a razão molar da IGF-1/IGFBP-3 têm demonstrado que doses habituais de hrGH elevam a razão molar em relação ao período pré-tratamento, porém ainda dentro de valores normais, sugerindo, assim, maior segurança do tratamento (16).

\section{CONSIDERAÇÕES FINAIS}

O tratamento com doses habituais do hrGH é seguro, com poucos eventos adversos sendo relatados, porquanto muitos desses não estão relacionados ao hrGH. No entanto, o acompanhamento desses pacientes até a vida adulta é crucial, especialmente daqueles em uso de doses farmacológicas ou daqueles que possuem condições de risco para complicações.

\section{REFERÊNCIAS}

1. Hintz LR. Untoward events in patients treated with growth hormone in the USA. Horm Res. 1992;38:44-9.

2. Bridges N. New indications for growth hormone. Arch Dis Child Educ Pract Ed. 2005;90:ep7-9.

3. Wilton P. Adverse events reported in KIGS. In: Ranke MB, Price DA, Reiter EO, editors. Growth hormone therapy in pediatrics - 20 years of KIGS. Basel: Karger; 2007.

4. Wyatt D. Lessons from the National Cooperative Growth Study. European Journal of Endocrinology. 2004;151:S55-9.

5. Rappaport R, Oberfield SE, Robison L, Salisbury S, David R, Rao J, et al. Relationship of growth hormone deficiency and leukemia. J Pediatr. 1995;126:759-61.

6. Ergun-Longmire B, Mertens A, Mitby P, Qin J, Heller G, Shi W, et al. Growth hormone treatment and risk of second neoplasm in the childhood cancer survivor. J Clin Endocrinol Metab. 2006;91:3494-8.

7. Growth Hormone Research Society. Consensus: critical evaluation of the safety of recombinant human growth hormone administration: statement from the Growth Hormone Research Society. J Clin Endocrinol Metab. 2001;86:1868-70.

8. Malazowsky S, Tanner L, Wysowsky D, Fleming G. Growth hormone, insulin-like growth factor 1 and benign intracranial hypertension. N Engl J Med. 1993;329:665-6.

9. Saenger P. Metabolic consequences of growth hormone treatment in paediatric practice. Horm Res. 2000;53 Suppl 1:60-9.

10. Chernausek SD, Backeljauw PF, Frane J, Kuntze J, Underwood LE. Long-term treatment with recombinant insulin-like growth factor (IGF)-1 in children with severe IGF-1 deficiency due to growth hormone insensitivity. J Clin Endocrinol Metab. 2007;92:902-10.

11. Kemp SF, Fowlkes JL, Thrailkill KM. Efficacy and safety of mecasermin rinfabate. Expert Opin Biol Ther. 2006 May;6(5):533-8.

12. Williams RM, McDonald A, O'Savage M, Dunger DB. Mecasermin rinfabate: rhIGF-I/rhlGFBP-3 complex: iPLEX. Expert Opin Drug Metab Toxicol. 2008;4(3):311-24.

13. Chan Jm, Stampfer MJ, Giovannucci E, Gann PH, Ma J, WiIkinson $\mathrm{P}$, et al. Plasma insulin-like growth factor 1 and prostate cancer risk: a prospective study. Science. 1998;279:563-6.

14. Burroughs KD, Dunn SE, Barrett JC, Taylor JA. Insulin-like growth factor-1: a key regulator of human cancer risk? J Natl Cancer Inst. 1999;91:579-81.

15. Park $P$, Cohen P. The role of insulin-like growth factor 1 monitoring in growth-hormone treated children. Horm Res. 2004;62 Suppl 1:59-65.

16. Scire G, Del Bianco C, Spadoni GL, Cianfarani S. Growth hormone therapy does not alter the insulin-like growth factor $1 /$ in sulin-like growth factor binding protein-3 molar ratio in growth hormone-deficient children. J Endocrinol Invest. 2008;31:153-8.

Endereço para correspondência:

Cristiane Kochi

Rua Madre Cabrini, 214, apto. 61, Vila Mariana

04020-000 São Paulo, SP

E-mail: ckochi@uol.com.br 